\title{
Influence of two updated nuclear reaction rates on the evolution of low and intermediate mass stars (Research Note)
}

\author{
A. Weiss ${ }^{1}$, A. Serenelli ${ }^{2,1}$, A. Kitsikis ${ }^{1}$, H. Schlattl ${ }^{1}$, and J. Christensen-Dalsgaard ${ }^{3}$ \\ 1 Max-Planck-Institut für Astrophysik, Karl-Schwarzschild-Str. 1, 85748 Garching, Germany \\ e-mail: weiss@mpa-garching.mpg.de \\ 2 Institute for Advanced Study, Einstein Drive, Princeton NJ 08540, USA \\ 3 Institut for Fysik og Astronomi, Aarhus Universitet, Bygn. 520, Ny Munkegade, 8000 Aarhus C, Denmark
}

Received 18 March 2005 / Accepted 19 July 2005

\section{ABSTRACT}

Two key reactions of hydrostatic nuclear burning in stars have recently been revised by new experimental data - the ${ }^{14} \mathrm{~N}(p, \gamma){ }^{15} \mathrm{O}$ and $3 \alpha$ reaction rates. We investigate how much the new rates influence the evolution of low-mass, metal-poor and metal-free stars and of an intermediatemass star of solar-type composition. We concentrate on phases of helium ignition or thermally unstable helium burning. Our global result is that the new $3 \alpha$ rate has no significant influence on such stars, but that there is a noticeable though small effect of the new ${ }^{14} \mathrm{~N}(p, \gamma){ }^{15} \mathrm{O}$ rate, in particular on the core helium flash and the blue loop during core helium burning in the intermediate-mass star.

Key words. stars: interiors - stars: evolution - nuclear reactions, nucleosynthesis, abundances

\section{Introduction}

The triple-alpha reaction is one of the key nuclear reactions for the synthesis of elements in stars and is also the main energy source during helium burning. The reaction rate is dominated by resonances, the best known being the one at $7.65 \mathrm{MeV}$, theoretically predicted by Hoyle (1954), but there is considerable interest in determining all resonances with high precision. Recently, Fynbo et al. (2005) reported new measurements concerning resonances of ${ }^{12} \mathrm{C}$ with $3 \alpha$-particles obtained from ${ }^{12} \mathrm{C}$-decay experiments. In particular, they found a dominant resonance at $\simeq 11 \mathrm{MeV}$, while they did not confirm another at 9.1 MeV reported previously (Angulo et al. 1999). The new reaction rate (called the "ISOL" rate in the following) deviates from the one published by Angulo et al. (1999, NACRE): at temperatures between $2.5 \times 10^{7}$ and $10^{8} \mathrm{~K}$ it is between 7 and $20 \%$ lower, but below $2.5 \times 10^{7} \mathrm{~K}$ up to $50 \%$ higher. The largest change occurs for $T>3 \times 10^{9} \mathrm{~K}$, where the new rate is lower by up to one order of magnitude (Fig. 1).

In the present paper we consider three cases of stellar evolution where a modified $3 \alpha$ rate might influence the models: the core helium flash in low-mass metal-poor stars, the core and shell helium burning in intermediate-mass stars of solar-like composition, and helium burning in metal-free low-mass stars. This mass range is interesting because of the high temperature sensitivity during core and shell flashes, where instabilities amplify even small temperature variations.

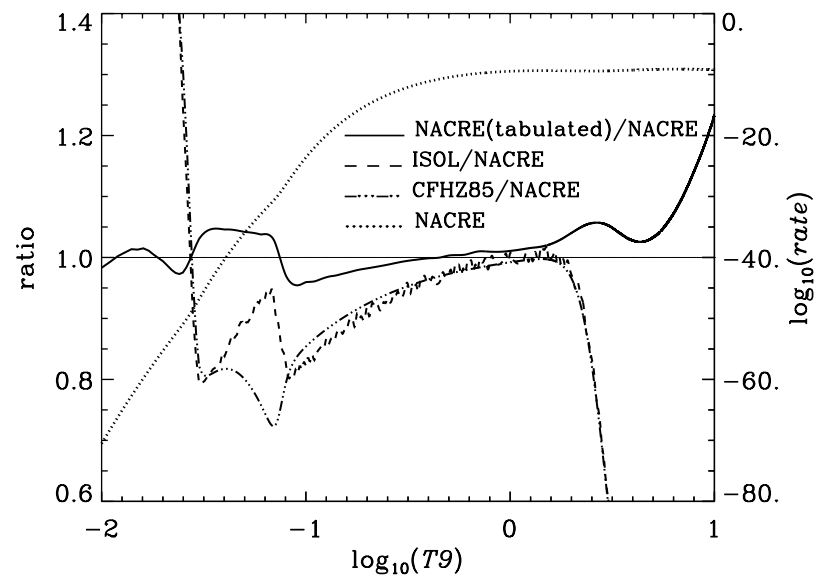

Fig. 1. $3 \alpha$ reaction rates relative to the NACRE analytic one (Angulo et al. 1999). The tabulated NACRE, CFHZ85 (Caughlan et al. 1985) and ISOL rates (Fynbo et al. 2005) are shown. The dotted line is the absolute value of the reference NACRE rate (scale on right axis).

Coincidentally, another key reaction has been redetermined recently by the LUNA collaboration, the ${ }^{14} \mathrm{~N}(p, \gamma){ }^{15} \mathrm{O}$ bottleneck reaction of the CNO-cycle (Formicola et al. 2004). The influence on globular cluster age determinations has already been discussed by Imbriani et al. (2004), and we will briefly comment on this. However, our main interest in this bottleneck reaction is how stars of low and intermediate mass might be influenced in their evolution. 
The outline of this paper is as follows: after describing briefly our stellar evolution code and the specific reaction rates for the $3 \alpha$ process and the ${ }^{14} \mathrm{~N}$ reaction in Sect. 2, we present the results for the three stellar evolution cases described above in Sect. 3, followed by the conclusion in Sect. 4 .

\section{Stellar evolution program}

\subsection{Basic properties}

All calculations were done with the Garching stellar evolution code as described by Weiss \& Schlattl (2000). The program has been modified in only minor aspects since then. The code incorporates the OPAL equation of state (Rogers et al. 1996) and the OPAL opacity tables (Iglesias \& Rogers 1996) supplemented by the molecular opacities of Alexander \& Ferguson (1994). In the calculations presented here, the equation of state (EOS) of Irwin (see Cassisi et al. 2003) has been used, which is based on the OPAL EOS. Microscopic diffusion of all elements is implemented and has been included in the calculations of Sect. 3.2. Convection is treated according to standard mixing length theory with the Schwarzschild criterion for stability. Mass loss is included according to Reimers' formula (Reimers 1975), in the generalized form (Iben \& Renzini 1983) containing a free scaling parameter $\eta$, which we will specify for each case.

Nuclear burning is taken into account by a network which treats hydrogen and helium burning separately, unless both protons and $\alpha$-particles are present and temperatures are high enough for helium processing. In such cases (see Sect. 3.2) the whole network and any mixing process are treated simultaneously. For details on this see Schlattl et al. (2001).

\section{2. ${ }^{14} \mathrm{~N}(p, \gamma)^{15} \mathrm{O}$ and $3 \alpha$ rate}

The standard implementation of the first rate $^{1}$ is according to the recommendation in Adelberger et al. (1998, Table VI; hereafter referred to as Ade198), with the astrophysical $S(0)$-factor being $3.5 \mathrm{keV}$ b. NACRE (Angulo et al. 1999) gives $S(0)=$ $3.2 \pm 0.8 \mathrm{keV}$ b. The new LUNA value (Formicola et al. 2004) is $1.7 \pm 0.1$ (stat) \pm 0.2 (sys) $\mathrm{keV}$ b; $S^{\prime}(0)$ and $S^{\prime \prime}(0)$ have been left unchanged at the Adelberger values and the new rate therefore differs from the old one by a constant factor. This is in agreement with the procedure of Imbriani et al. (2004).

The standard implementation of the $3 \alpha$ rate in our program is the analytic form of Caughlan et al. (1985, CFHZ85). Since Fynbo et al. (2005) used the analytic fit to the NACRE rate for reference, we implemented the same rate for the comparisons. The new ISOL rate was available to us in tabulated form (Chr. Diget, private communication). Figure 1 shows all $3 \alpha$ rates compared with the NACRE analytic rate. Note that in the temperature range of interest to us the new rate lies within the uncertainty of the NACRE rate (Angulo et al. 1999) and that the analytic fit of the NACRE rate deviates from the tabulated values by up to $5 \%$. The ISOL rate agrees quite well with that

\footnotetext{
${ }^{1}$ The term "rate" is to be understood as $N_{A}^{(n-1)}\langle\sigma v\rangle$, that is as the Maxwellian-averaged reaction rate (see Angulo et al. 1999) in units of $\mathrm{cm}^{3} \mathrm{~mol}^{-1} \mathrm{~s}^{-1}$ for $n$ reaction partners.
}

of Caughlan et al. (1985) over an extended range below temperatures of several billion degrees. The critical temperature above which helium burning is taken into account was set to $5 \times 10^{7} \mathrm{~K}$; using still lower values has no influence on the models.

\section{Sample calculations}

\subsection{Core helium flash in a Pop. II star}

In this section we present the results of our sample calculations and compare them with reference calculations that use the NACRE and Adel 98 rates for the $3 \alpha$ and ${ }^{14} \mathrm{~N}(p, \gamma){ }^{15} \mathrm{O}$ reaction rates, respectively. We begin with the onset of helium burning in stars of low mass and degenerate helium cores, the so-called core helium flash. We performed calculations for two masses, $0.8 M_{\odot}$ and $1.0 M_{\odot}$, and three metallicities, $Z=$ $0.001,0.0001,0.00001$ and initial helium content $Y=0.25$. No mass loss was assumed. The calculations were started from the zero-age main sequence (ZAMS) and followed through the flash until the star settles on the horizontal branch (HB) and then up to the early AGB.

Updating the $3 \alpha$ rate, the changes are very small but as would be expected from a slightly lower rate. The tip of the RGB is almost unchanged, the luminosity being $2 \%$ higher for the new rate. The core mass increases very little, between 0.002 and $0.003 M_{\odot}$ depending on mass and metallicity. The age of the models also increases by a mere $0.2 \mathrm{Myr}$ at most. We show the resulting tracks in the Hertzsprung-Russell Diagram (HRD) in Fig. 2 for the lowest metallicity and mass, and list a few quantities in Table 1. Note that even the secondary flashes during the settling to the HB are almost indistinguishable. The agreement between the two cases is as close for all other cases.

We then varied the $\left.{ }^{14} \mathrm{~N}(p, \gamma)\right)^{15} \mathrm{O}$ rate $(3 \alpha=\mathrm{NACRE})$. The effect turned out to be much larger, and can be seen in Fig. 2 (lower panel). We notice differences already around the turnoff, and in particular during the approach to the HB; the effective temperature of the zero-age HB model increases by up to $20 \%$ for the LUNA rate.

Imbriani et al. (2004) showed that due to the improved ${ }^{14} \mathrm{~N}(p, \gamma){ }^{15} \mathrm{O}$ rate the ages of globular clusters increase by up to 1 Gyr. This is not simply due to the slower bottleneck reaction leading to longer timescale for hydrogen fusion. In Fig. 3 we display for one example $\left(1 M_{\odot}, Z=0.001\right)$ the evolution of luminosity and effective temperature during main-sequence and red-giant-branch evolution. Evidently, the luminosity evolution on the main sequence is hardly affected by the nuclear rate. Energy generation takes place within a core that has a temperature only marginally higher but is more extended as the result of a shallower temperature profile. So, while at the center hydrogen is burning at a slower pace (same $T$, lower reaction rate), more mass is involved in the nuclear fusion. The time it takes to deplete hydrogen in the core is therefore somewhat higher (in our case $+190 \mathrm{Myr}$ ), but the exhausted core is larger $\left(+0.013 M_{\odot}\right)$. Due to that larger core mass, towards the end of the main sequence the central temperature is higher by approximately 5\% for the LUNA rate. Another consequence of the different core evolution is a modified temperature gradient, and thus $T_{\text {eff }}$ at the end of the main sequence evolves differently 

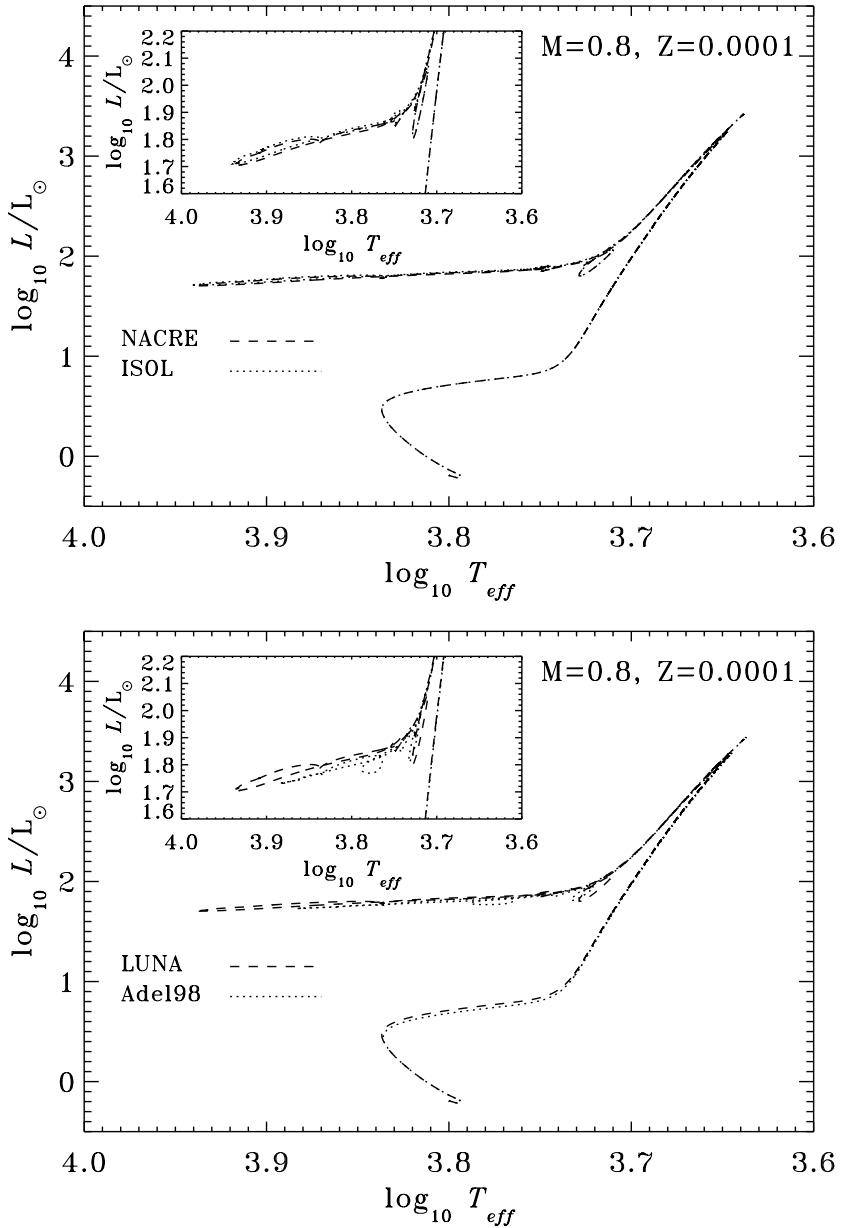

Fig. 2. Evolution of a stellar model of $0.8 M_{\odot}$ and $Z=10^{-4}$ from ZAMS to HB. In the upper panel we varied the $3 \alpha$ - and in the lower one the ${ }^{14} \mathrm{~N}(p, \gamma){ }^{15} \mathrm{O}$-rate. The insets show the approach to the HB in greater detail.

(Fig. 3), influencing the turn-off luminosity which is defined as the value of $L$ at the bluest point along the MS track. To be more quantitative, the turn-off in the case of the Adelberger rate is at $t=4.38 \mathrm{Gyr}, \log T_{\text {eff }}=3.852$, and $\log L / L_{\odot}=0.554$. For the revised LUNA rate the hottest point is reached later, at $\log T_{\text {eff }}=3.854$, when $\log L / L_{\odot}=0.593$ and $t=4.62 \mathrm{Gyr}$, an increase of $240 \mathrm{Myr}$. Note, however, that at the turn-off age of the Adelberger case, $T_{\text {eff }}=3.854$ and $\log L / L_{\odot}=0.558$ for LUNA, i.e., almost identical. Thus, the change in the turn-off is due to the modified interior structure, which influences the morphology of the evolutionary track. We have also investigated a few other cases and find that at even lower metallicity $(Z=0.0001)$ the age differences decrease to $1-2 \%$.

\subsection{Core helium flash in a Pop. III star}

A particularly interesting variant of the core helium flash is that found in metal-free (Pop. III) stars. Details on this subject were provided by Weiss et al. (2000) and Schlattl et al. (2001). We have calculated the evolution of a star of $M=1 M_{\odot}$, $Y_{i}=0.23(Z=0)$. We varied both rates as before, the reference case using the Adelberger et al. (1998) ${ }^{14} \mathrm{~N}(p, \gamma){ }^{15} \mathrm{O}$ and
Table 1. Selected properties of one of our Pop. II models $(M=$ $0.8 M_{\odot} ; Z=10^{-4}$ ) at critical stages of its evolution from ZAMS to ZAHB evolved with various combinations of reaction rates as shown in Fig. 2. $M_{\mathrm{He}}$ is the helium core mass in solar unit at the helium flash.

\begin{tabular}{ccccc}
\hline \hline${ }^{14} \mathrm{~N}+\mathrm{p}:$ & & Ade198 & \multicolumn{2}{c}{ LUNA } \\
\hline $3 \alpha:$ & & NACRE & NACRE & ISOL \\
\hline \multirow{3}{*}{ Turn off } & $\log L / L_{\odot}$ & 0.437 & 0.467 & 0.467 \\
& $\log T_{\text {eff }}$ & 3.8360 & 3.8368 & 3.8368 \\
& $\operatorname{Age}[\mathrm{Myr}]$ & 11130 & 11273 & 11273 \\
\hline \multirow{4}{*}{ RGB-tip } & $\log L / L_{\odot}$ & 3.285 & 3.237 & 3.246 \\
& $\log T_{\text {eff }}$ & 3.6449 & 3.6479 & 3.6474 \\
& $M_{\mathrm{He}}$ & 0.4990 & 0.5052 & 0.5074 \\
& $\operatorname{Age}[\mathrm{Myr}]$ & 12638 & 12699 & 12699 \\
\hline \multirow{2}{*}{ ZAHB } & $\log L / L_{\odot}$ & 1.744 & 1.715 & 1.722 \\
& $\log T_{\text {eff }}$ & 3.8614 & 3.9324 & 3.9344 \\
\hline
\end{tabular}

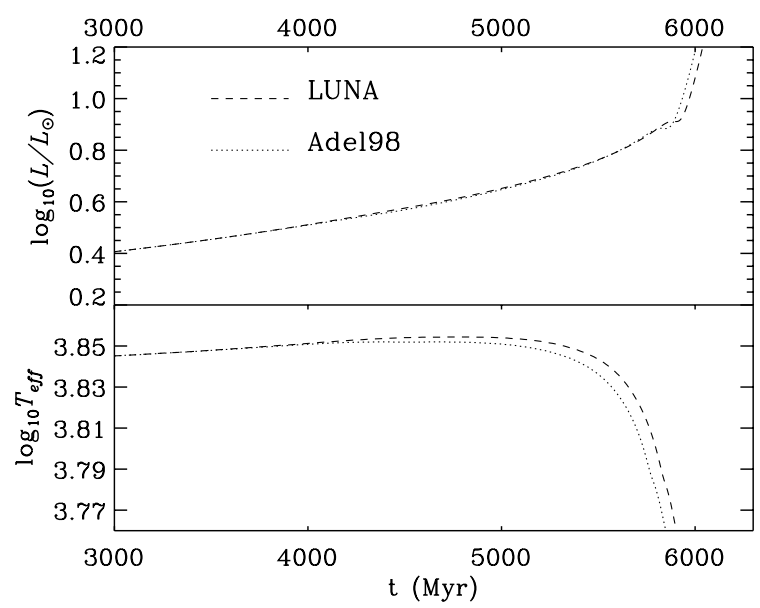

Fig. 3. Comparison of luminosity and effective temperature as functions of time (in Myr) for a star with $1 M_{\odot}$ and $Z=0.001$, calculated with both the Adelberger (dotted line) and LUNA (dashed line) ${ }^{14} \mathrm{~N}(p, \gamma){ }^{15} \mathrm{O}$ rate.

NACRE $3 \alpha$ rates. Some properties of the models are listed in Table 2. We also investigated the case "LUNA + CFHZ85", but do not discuss it further, because it is indistinguishable from the "LUNA + ISOL" case.

The first particularity of Pop. III evolution is a loop in the HRD after the main sequence, which is due to spurious carbon production in the hot core and the ignition of the CNO-cycle (for details see Weiss et al. 2000). The presence of this loop depends on the stellar mass; it is present in our reference case, but vanishes in all cases with the new (lower) LUNA rate for ${ }^{14} \mathrm{~N}(p, \gamma){ }^{15} \mathrm{O}$. As the reason for the disappearance of the loop we identify the fact that the lower CNO-luminosity prevents the creation of a convective core, which otherwise leads to an increase of the hydrogen abundance in the core and thus to a luminosity enhancement. We note that the creation of the first insitu carbon nuclei is not influenced by the choice of the $3 \alpha$ rate.

The second particular event is the (first) core helium flash that happens at a much lower luminosity in Pop. III stars than in those with $Z \gtrsim 10^{-6}$, due to the higher core temperature. As the H-shell temperatures are higher as well, the so-called entropy barrier is lower and a mixing event between the hydrogen-rich 
Table 2. Selected properties of Pop. III models evolved with various combinations of reaction rates. We list luminosity and helium core mass at the onset of the first and second core helium flash, as well as the maximum helium luminosity $\left(\log L_{\mathrm{He}}^{\max } / L_{\odot}\right)$ and the mass at which the maximum temperature is reached $\left(M_{\mathrm{T}_{\max }} / M_{\odot}\right)$ at the time of $\log L_{\mathrm{He}}^{\max } / L_{\odot}$. In addition, the surface abundances (mass fractions) after the flash-induced mixing event is given for key elements.

\begin{tabular}{llccc}
\hline \hline${ }^{14} \mathrm{~N}+\mathrm{p}$ & & Adel98 & \multicolumn{2}{c}{ LUNA } \\
\hline $3 \alpha$ & & NACRE & NACRE & ISOL \\
\hline MS & $\mathrm{CN}-\operatorname{loop}$ & yes & no & no \\
\hline \multirow{4}{*}{ 1st flash } & $\log \left(L / L_{\odot}\right)$ & 2.324 & 2.302 & 2.311 \\
& $\log \left(L_{\mathrm{He}}^{\max } / L_{\odot}\right)$ & 10.08 & 10.01 & 10.03 \\
& $M_{\mathrm{He}} / M_{\odot}$ & 0.4784 & 0.4755 & 0.4780 \\
& $M_{\mathrm{T}_{\max }} / M_{\odot}$ & 0.1849 & 0.1818 & 0.1863 \\
\hline \multirow{4}{*}{ dredge up } & $\mathrm{H}$ & 0.5288 & 0.5369 & 0.5366 \\
surface & $\mathrm{He}$ & 0.4582 & 0.4510 & 0.4510 \\
abdcs. & ${ }^{12} \mathrm{C}$ & 0.00417 & 0.00292 & 0.00302 \\
& ${ }^{14} \mathrm{~N}$ & 0.00111 & 0.00080 & 0.00083 \\
& ${ }^{15} \mathrm{~N}$ & 0.00772 & 0.00837 & 0.00861 \\
& ${ }^{16} \mathrm{O}$ & $2.6 \times 10^{-7}$ & $1.1 \times 10^{-7}$ & $1.1 \times 10^{-7}$ \\
& $\log \left(L / L_{\odot}\right)$ & $3.9 \times 10^{-6}$ & $3.4 \times 10^{-6}$ & $4.0 \times 10^{-6}$ \\
\hline \multirow{4}{*}{ 2nd flash } & $\log \left(L_{\mathrm{He}}^{\max } / L_{\odot}\right)$ & 9.532 & 3.487 & 3.498 \\
& $M_{\mathrm{He}} / M_{\odot}$ & 0.4585 & 9.764 & 9.770 \\
& $M_{\mathrm{T}_{\max }} / M_{\odot}$ & 0.0789 & 0.4615 & 0.4638 \\
& & & & 0.0729 \\
\hline
\end{tabular}

envelope and the hot carbon-enriched helium layers is possible (see also Fujimoto et al. 2000). As a consequence, the envelope will be enriched drastically in CNO-products and helium (see Table 2). As in the case of ordinary Pop. II stars, the new $3 \alpha$ rate has only a very moderate influence on the flash: it starts at slightly higher luminosity and core mass, but the increase is not significant. The peak helium luminosity $L_{\mathrm{He}}^{\max }$ is unchanged, within the computational uncertainties.

In contrast, the new ${ }^{14} \mathrm{~N}(p, \gamma){ }^{15} \mathrm{O}$ rate has a much stronger influence. The flash luminosity is lower due to the higher shell temperatures and the core mass is higher. This is probably a consequence of the fact that the core mass-luminosity relation depends on the temperature exponent of the shell hydrogen burning. For CNO-burning, it is $L \propto M_{c}^{7}$, and for $p p$-burning $\propto M_{c}^{3}$. Very metal-poor stars have a very small contribution from the CNO-cycle only and therefore follow closely the latter relation. A lower ${ }^{14} \mathrm{~N}(p, \gamma){ }^{15} \mathrm{O}$ rate leads to a smaller temperature exponent, and thus the core mass is slightly higher at given luminosity.

The result of the flash-induced dredge-up shows larger (relative) variations (Table 2), but with no apparent systematics, except that in the table the dredge-up appears generally to be stronger for the older rates, while for the latest rate more CN-burning could take place.

After the star has settled again on the giant branch, it resumes its evolution since core helium burning has been extinguished as a result of the first flash and mixing. A second, more moderate flash appears at a standard RGB tip luminosity $\left(\log L / L_{\odot} \approx 3.5\right)$. Table 2 demonstrates again that the largest changes are due to the new LUNA ${ }^{14} \mathrm{~N}(p, \gamma){ }^{15} \mathrm{O}$ rate, leading to lower ignition luminosity but higher helium core mass. The largest variation found in Table 2 is therefore between the

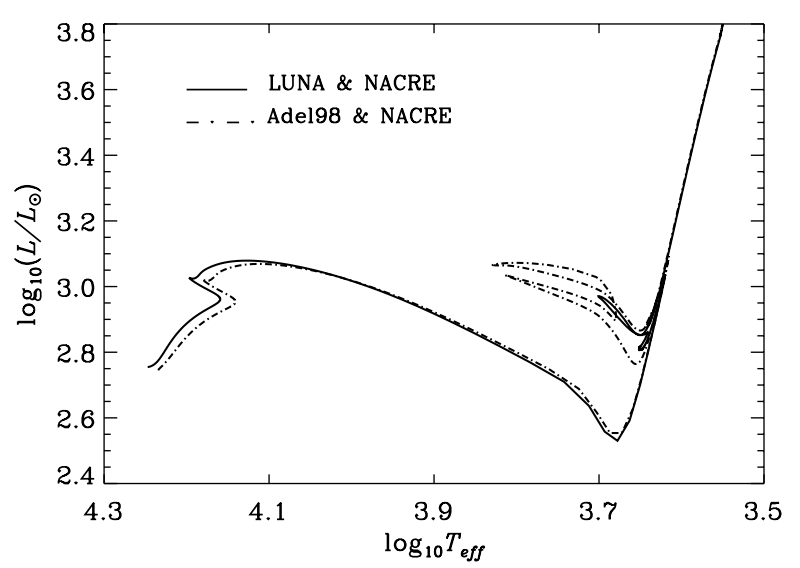

Fig. 4. Evolution in the HRD for the Pop. I model with $M=5 M_{\odot}$, showing the influence of changing the ${ }^{14} \mathrm{~N}(p, \gamma){ }^{15} \mathrm{O}$ reaction rate. The two cases are indicated in the figure.

reference case (Col. 3) and that updating the ${ }^{14} \mathrm{~N}(p, \gamma){ }^{15} \mathrm{O}$ rate only (Col. 4).

\subsection{Helium burning and thermal pulses in a Pop. I star}

As a third case we have investigated that of a typical solarmetallicity intermediate-mass star evolving into and through the Asymptotic Giant Branch (AGB) phase. The composition of our model was $X=0.695, Y=0.285$ and $Z=0.02$. Mass loss according to Reimers was taken into account with the scaling parameter $\eta$ being 0.4 on the RGB and 0.5 on the AGB.

In Fig. 4 we show the evolution of this model in the HRD for the two $\left.{ }^{14} \mathrm{~N}(p, \gamma)\right)^{15} \mathrm{O}$ reaction rates. Using the updated LUNA rate the main-sequence evolution takes place at higher temperatures, implying a more compact structure of the model. This is similar to the Pop. II model (Sect. 3.1) but more pronounced. As the evolution of intermediate mass stars is very sensitive to the internal composition profile, the consequences for the core helium burning phase are quite large: the blue loop gets significantly shorter.

The luminosity variations during the thermal pulses (TP) along the AGB are shown in Fig. 5. The TPs start earlier by about 1 million years for the LUNA rate, which is due to a shorter main-sequence lifetime. We have therefore shifted the time axis for the Ade198 case to coincide at the pre-flash luminosity maximum. The pulse behaviour is similar, although the peak luminosity and peak helium luminosity (not shown) are higher, and the interpulse duration longer for the new LUNA rate. This is consistent with earlier findings concerning the influence of H-burning rates on pulse behaviour (Despain \& Scalo 1976), and in particular with the recent investigation by Herwig \& Austin (2004), who found stronger flashes for lower ${ }^{14} \mathrm{~N}(p, \gamma){ }^{15} \mathrm{O}$ rates in a $2 M_{\odot}$ model. These differences become smaller with increasing pulse number. We followed 15 (LUNA) respectively 8 (Ade198) pulses and then stopped the calculations.

Using the new LUNA ${ }^{14} \mathrm{~N}(p, \gamma){ }^{15} \mathrm{O}$ reaction rate, we then compared models calculated with the three variants of the $3 \alpha$ rate. Again, the blue loops, being most sensitive to structure variations, show the clearest reaction, and get even shorter for 


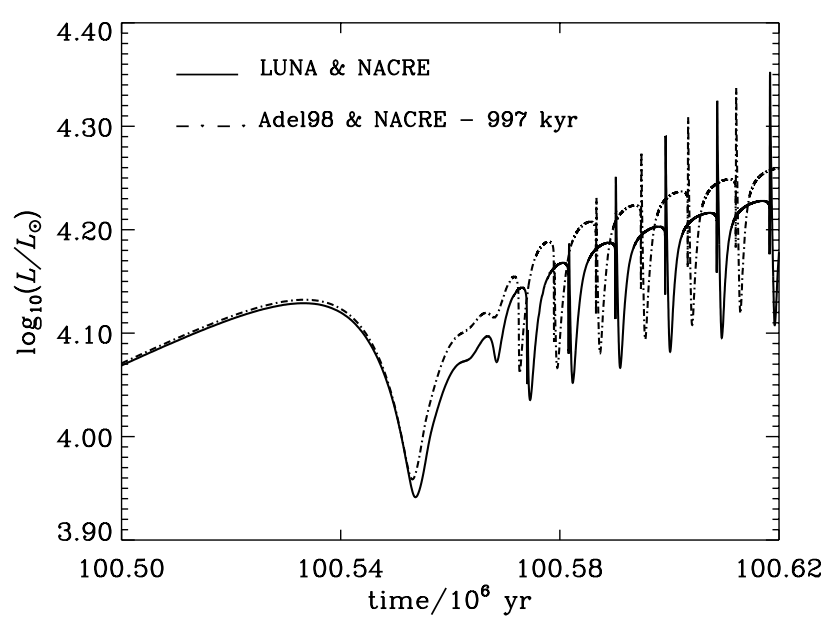

Fig. 5. Thermal pulses on the AGB for two different ${ }^{14} \mathrm{~N}(p, \gamma)^{15} \mathrm{O}$ reaction rates. Shown is the total luminosity as function of time (in million years). To display better the differences the time axis for the Adel98 ${ }^{14} \mathrm{~N}(p, \gamma){ }^{15} \mathrm{O}$ case has been shifted by $-997 \mathrm{kyr}$.

the new ISOL rate. Concerning the thermal pulses, they are extremely similar to each other, and if shifted by $-80 \mathrm{kyr}$ for the CFHZ85 and $-67 \mathrm{kyr}$ for the new ISOL $3 \alpha$ rate relative to the NACRE case they completely agree.

Finally, we mention the chemical composition of all cases investigated in terms of central carbon and oxygen abundance after core helium burning. The carbon abundance ranges from 0.200 to 0.223 with the highest value reached for the older reaction rates. Consequently the core most rich in oxygen is obtained for the LUNA ${ }^{14} \mathrm{~N}(p, \gamma){ }^{15} \mathrm{O}$ and new $3 \alpha$ rates combined. However, in spite of noticeable variations, they are minor compared with the uncertainties still present due to the ${ }^{12} \mathrm{C}(\alpha, \gamma){ }^{16} \mathrm{O}$ rate. As an example, we quote here the result of Bono et al. (2000,their Table 6) that the time spent in the blue loop by a $5 M_{\odot}$ star changes by up to $2.12 \mathrm{Myr}(33 \%)$, the mass of the CO-core by $0.26 M_{\odot}(5 \%)$, and the $\mathrm{C} / \mathrm{O}$ ratio by a factor of 3 when varying the rate within a factor of 2.3 , which is comparable to the rate uncertainty (Kunz et al. 2002).

\section{Conclusions}

We have investigated the influence of new rates for the ${ }^{14} \mathrm{~N}(p, \gamma){ }^{15} \mathrm{O}$ and $3 \alpha$ reactions on the evolution of low- and intermediate-mass stars, considering cases in which helium burning proceeds under thermally unstable conditions. In all cases we find negligible changes in the evolution and also in the interior evolution due to the new $3 \alpha$ rate. The RGB tip brightness is slightly increased due to the lower rate at very low temperatures corresponding to the earliest phases of helium ignition. The largest effect shows up in the blue loops during core helium burning of the $5 M_{\odot}$ star, emphasizing the sensitivity of these loops to details of the interior structure.
The ${ }^{14} \mathrm{~N}(p, \gamma){ }^{15} \mathrm{O}$ rate has a definitely stronger influence. It prolongs the duration of central hydrogen burning, increases the turn-off temperature and thus indirectly the turn-off location, it leads to a disappearance of the $\mathrm{CN}$ flash in the Pop. III post-main-sequence star, strongly reduces the blue loop during core helium burning of a $5 M_{\odot}$ star, and also influences the thermal pulses. Interestingly, these are, apart from a very minor shift in time, almost identical in the case of varying the $3 \alpha$ rate.

We conclude that the new $3 \alpha$ rate has no influence on the evolution of low and intermediate-mass stars, and that the effect of the LUNA ${ }^{14} \mathrm{~N}(p, \gamma)^{15} \mathrm{O}$ is tiny, but noticeable. Further investigations into its effect on more massive stars as well as of the effect of the $3 \alpha$ rate on massive stars are needed. If the differences in the various rate determinations we have used are representative of the experimental errors, then both rates are no longer a source of uncertainty for stellar modeling.

Acknowledgements. We thank Chr. Diget for making available the rate table. F. Meissner kindly provided data for the turn-off values of metal-poor stars, and F. Herwig information about his AGB-models with the new rates. A.W. is grateful to L. Girardi for explanations concerning the core mass-luminosity relation. A.M.S. is supported in part by NSF grant PHY-0070928.

\section{References}

Adelberger, E., Austin, S., Bahcall, J., et al. 1998, Rev. Mod. Phys., 70,1265

Alexander, D. R., \& Ferguson, J. W. 1994, ApJ, 437, 879

Angulo, C., Arnould, M., Rayet, M., et al. 1999, Nucl. Phys. A, 656, 3

Bono, G., Caputo, F., Cassisi, S., et al. 2000, ApJ, 543, 955

Cassisi, S., Salaris, M., \& Irwin, A. 2003, ApJ, 588, 862

Caughlan, G., Fowler, W., Harris, H., \& Zimmerman, B. 1985, Atomic Data and Nuclear Data Tables, 32, 197

Despain, K., \& Scalo, J. 1976, ApJ, 208, 789

Formicola, A., Imbriani, G., Costantini, H., et al. 2004, Phys. Lett. B, 591, 61

Fujimoto, M. Y., Ikeda, Y., \& Iben, I. 2000, ApJ, 529, L25

Fynbo, H., Diget, C., Bergemann, U., et al. 2005, Nature, 433, 136

Herwig, F., \& Austin, S. 2004, ApJ, 613, L73

Hoyle, F. 1954, ApJS, 1, 121

Iben, I., \& Renzini, A. 1983, ARA\&A, 21, 271

Iglesias, C. A., \& Rogers, F. J. 1996, ApJ, 464, 943

Imbriani, G., Costantini, H., Formicola, A., et al. 2004, A\&A, 420, 625

Kunz, R., Fey, M., Jaeger, M., et al. 2002, ApJ, 567, 643

Reimers, D. 1975, Mem. Soc. Roy. Sci. Liège, 8, 369

Rogers, F. J., Swenson, F. J., \& Iglesias, C. A. 1996, ApJ, 456, 902

Schlattl, H., Cassisi, S., Salaris, M., \& Weiss, A. 2001, ApJ, 559, 1082

Weiss, A., Cassisi, S., Schlattl, H., \& Salaris, M. 2000, ApJ, 533, 413

Weiss, A., \& Schlattl, H. 2000, A\&AS, 144, 487 